\title{
$N+1$ redundancy on ATCA instrumentation for Nuclear Fusion
}

\author{
Miguel Correia $^{\mathrm{a}, *}$, Jorge Sousa ${ }^{\mathrm{a}}$, António P. Rodrigues ${ }^{\mathrm{a}}$, António J.N. Batista ${ }^{\mathrm{a}}$, \\ Álvaro Combo a , Bernardo B. Carvalho ${ }^{a}$, Bruno Santos ${ }^{a}$, Paulo F. Carvalho ${ }^{a}$, \\ Bruno Gonçalves $^{a}$, Carlos M.B.A. Correia ${ }^{b}$, Carlos A.F. Varandas ${ }^{a}$ \\ a Associação EURATOM/IST, Instituto de Plasmas e Fusão Nuclear, Instituto Superior Técnico - Universidade Técnica de Lisboa, Lisboa, Portugal \\ ${ }^{\mathrm{b}}$ Centro de Instrumentação, Departamento de Física, Universidade de Coimbra, Coimbra, Portugal
}

\section{H I G H L I G H T S}

- In Nuclear Fusion, demanding security and high-availability requirements call for redundancy to be available.

- ATCA standard features desirable redundancy features for Fusion instrumentation.

- The developed control and data acquisition hardware modules support additional redundancy schemes.

- Implementation of $N+1$ redundancy of host processor and I/O data modules.

\section{A R T I C L E I N F O}

\section{Article history:}

Available online 19 February 2013

Keywords:
ATCA
AMC
PCIe
Redundancy
HA

HA

\begin{abstract}
A B S T R A C T
The role of redundancy on control and data acquisition systems has gained a significant importance in the case of Nuclear Fusion, as demanding security and high-availability requirements call for redundancy to be available. IPFN's control and data acquisition system hardware is based on an Advanced Telecommunications Computing Architecture (ATCA) set of I/O (DAC/ADC endpoints) and data/timing switch modules, which handle data and timing from all $\mathrm{I} / \mathrm{O}$ endpoints. Modules communicate through Peripheral Component Interconnect Express (PCle), established over the ATCA backplane and controlled by one or more external hosts. The developed hardware modules were designed to take advantage of ATCA specification's redundancy features, namely at the hardware management level, including support of: (i) multiple host operation with $N+1$ redundancy - in which a designated failover host takes over data previously assigned to a suddenly malfunctioning host and (ii) $N+1$ redundancy of $\mathrm{I} / \mathrm{O}$ and data/timing switch modules. This paper briefly describes IPFN's control and data acquisition system, which is being developed for ITER fast plant system controller (FPSC), and analyses the hardware implementation of its supported redundancy features.
\end{abstract}

(C) 2013 Elsevier B.V. All rights reserved.

\section{Introduction}

In advanced, large-scale Physics experiments, such as those of Nuclear Fusion devices, safety of operation is a critical issue, affecting equipment, facilities, personnel and environment. These are often highly complex systems and the malfunction of a single component may originate relevant damage to the entire infrastructure. Therefore, control subsystems envisaged for recent and forthcoming Physics experiment devices are required to ensure safety and reliability. This usually includes fault-tolerance features to automatically mitigate possible failures while still maintaining a stable and satisfactory performance [1]. Fault-tolerance also benefits the system's availability - of great importance in the case of Fusion

\footnotetext{
* Corresponding author. Tel.: +351239410108.

E-mail address: miguelfc@ipfn.ist.utl.pt (M. Correia).
}

devices as the increasing duration of plasma discharges, toward steady-state operation, calls for high-availability (HA). The socalled "five nines" (99.999\%) of up-time, which correspond to about 5 min of down-time per year, are often used as reference for HA requirements [2].

Fault-tolerant systems are directly linked with redundancy capabilities - having more than one resource to perform a certain task. Redundant resources are managed by the system to establish the desired level of functionality, compensating for eventual failures.

The Advanced Telecommunications Computing Architecture (ATCA) [3] was the instrumentation standard chosen for developing IPFN's control and data acquisition system hardware for Fusion experiments, having already delivered successful results in previous versions $[4,5]$. Besides being the most adequate for achieving real-time MIMO control [6], ATCA is inherently fault tolerant, with redundant power, shelf management and backplane interconnect. 
This particular ATCA-based control and data acquisition subsystem is part of the ITER fast plant system controller (FPSC) prototyping program, whose specification will be applicable to several diagnostic systems targeting plasma control [7].

The developed hardware modules use upcoming "xTCA for Physics" extensions [8]. These ATCA specification extensions introduce dedicated features for instrumentation in Physics, which promise to improve system performance and, through standardization, facilitate hardware development. The system comprises a set of I/O (DAC/ADC endpoints) and data/timing switch modules, which handle data and timing from all $\mathrm{I} / \mathrm{O}$ endpoints. Modules communicate through Peripheral Component Interconnect Express (PCle) network [9], established over the ATCA backplane and controlled by one or more external hosts.

The developed hardware modules were designed to take advantage of ATCA specification's redundancy features, namely at the hardware management level, including support of: (i) multiple host operation with $N+1$ redundancy - in which a designated failover host takes over data previously assigned to a suddenly malfunctioning host and (ii) $N+1$ redundancy of $\mathrm{I} / \mathrm{O}$ and data/timing switch modules.

This paper will describe the developed control and data acquisition system, and present the implementation of redundancy features in order to further enhance the system's availability possibilities.

\section{ATCA instrumentation}

The control and data acquisition subsystem is composed of ATCA instrumentation modules residing in an ATCA shelf, with respective power and shelf management units. The shelf is populated with two types of boards: (i) ATCA-PTSW-AMC4 - a data and timing switch/hub carrying up to four AMC modules [10] and (ii) ATCA-IO-PROCESSOR - node board containing the I/O (DAC and/or $A D C)$ endpoints [11]. The main system connections are shown in Fig. 1.
Two ATCA-PTSW-AMC4 modules will occupy hub positions (logical slots 1 and 2 ) of a standard ATCA shelf. These boards switch PCle data from compliant node boards (ATCA-IO-PROCESSOR) to the host computer, through the fabric interface. This topology is denominated dual-star, where each hub forms a (single) star connecting to all nodes.

PCle switching is provided by a PLX Technology ${ }^{\circledR}$ PEX $8696 \mathrm{PCI}$ Express Gen 2 Multi-Root switch, capable with 24 configurable ports, on-chip Non-Transparent port for dual-host and fail-over applications and hot-plug support [12]. In the ATCA-PTSW-AMC4 application, the PEX 8696 switches data from/to: (i) the four AMC slots $(4 \times$ link on each AMC); (ii) the rear transmission module (RTM) interface (16× link); and (iii) the fabric interface channels ( $1 \times$ link for each of the 13 channels connected). Any of these links can be selected as the upstream port, where the host computer is connected. IPFN also developed an RTM to interface to an external PCle host.

The ATCA-PTSW-AMC4 also serves as a timing distribution unit, implemented on a Virtex-6 Field Programmable Gate Array (FPGA) [13], which interfaces ATCA clocks with the AMC and RTM timing signals. ATCA clocks are spread to all node boards through the backplane's Clock and Synchronization interface.

The ATCA standard specifies a communication interface dedicated to hardware management - Intelligent Platform Management Interface (IPMI). Therefore all ATCA modules contain an Intelligent Platform Management Controller (IPMC), which communicates with the ATCA Shelf Manager to execute hardware management tasks [14].

\section{Redundancy features}

To achieve the desired levels of availability, the ATCA specification includes several redundancy features, starting with the -48 VDC dual-redundant power feeds of the shelf. The cooling system is also redundant. The Shelf Manager is responsible for management of all hardware resources and may also have a redundant spare. The

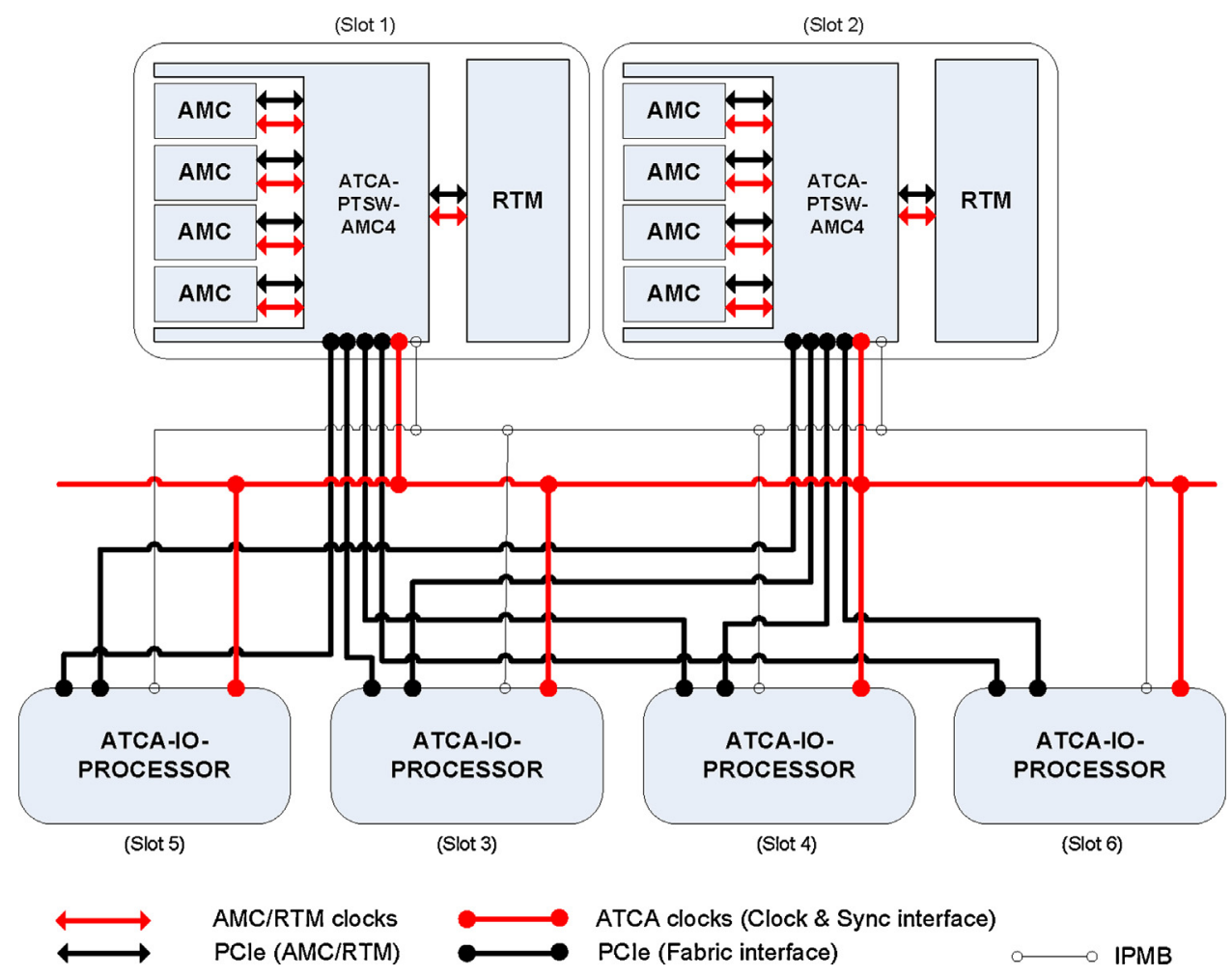

Fig. 1. The control and data acquisition subsystem: hardware modules and networks established on the ATCA shelf. 


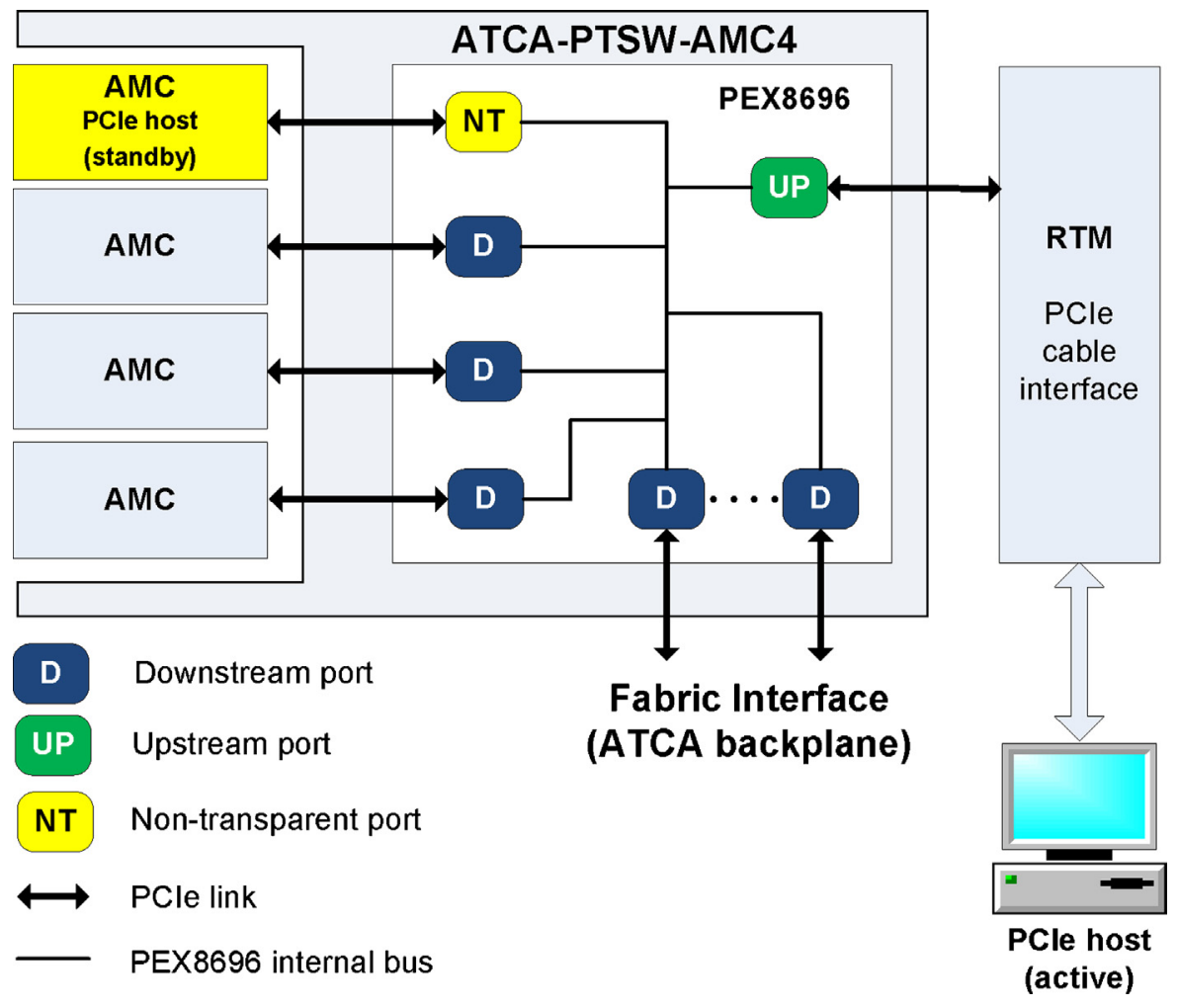

Fig. 2. Active-standby “ $1+1$ ” redundant host scheme.

Shelf Manager Controllers communicate with the remaining IPMCs through a redundant dedicated data bus (IPMB-0).

The backplane of the ATCA shelf provides high-speed serial links which can be used in various configuration topologies such as star (one hub connecting multiple nodes), dual-star (star with added redundant hub), dual dual-star (two distinct dual-stars for distinct purposes) or full-mesh, where each board acts simultaneously as a hub or node (direct data path from each board to the remaining boards in the system). All ones, with which board redundancy schemes can be implemented.

The present application utilizes dual-star topology on the fabric channel (PCle links). The two ATCA-PTSW-AMC4 modules occupy (hub) slots 1 and 2 of the shelf. Both connect to each of the node modules and interconnect each other. This topology allows several redundancy schemes to be implemented.

\subsection{Redundant PCle host processors}

Each of the two ATCA-PTSW-AMC4 hub boards PCle network can be hosted by either an external computer, using the RTM external cabling interface, or using an AMC host in any of the available slots.
The PEX8696 can configure any of these devices as the PCle host, selecting the correspondent data port as the upstream port. However, the PEX8696 allows a spare host, connected to a selectable non-transparent (NT) port, to be in standby mode. Upon a detected fault on the active host, the spare host is entitled to stand in for the faulty host by swapping their PEX8696 port configuration types from "upstream" to "non-transparent" and vice versa. Fig. 2 is an example using the ATCA-PTSW-AMC4 board, having an external RTM active host and an AMC host as spare to achieve board-level " $1+1$ " redundancy.

Another possible host-redundancy scenario is presented in Fig. 3. Both hub boards and respective PCle hosts are simultaneously in active mode. NT ports in each hub interconnect the two hosts using the Fabric Interface. Initially, each host processes data from its own downstream ports. If one host fails, it is detected by the other host via NT port. Data ports left by the faulty host are assigned to the healthy host, which configures its NT port as downstream and the faulty host device's NT port as upstream.

The previous models use a single active host per device. We now analyze the possibility of having multiple (active or standby) hosts connected to a single hub board using PEX8696's Virtual

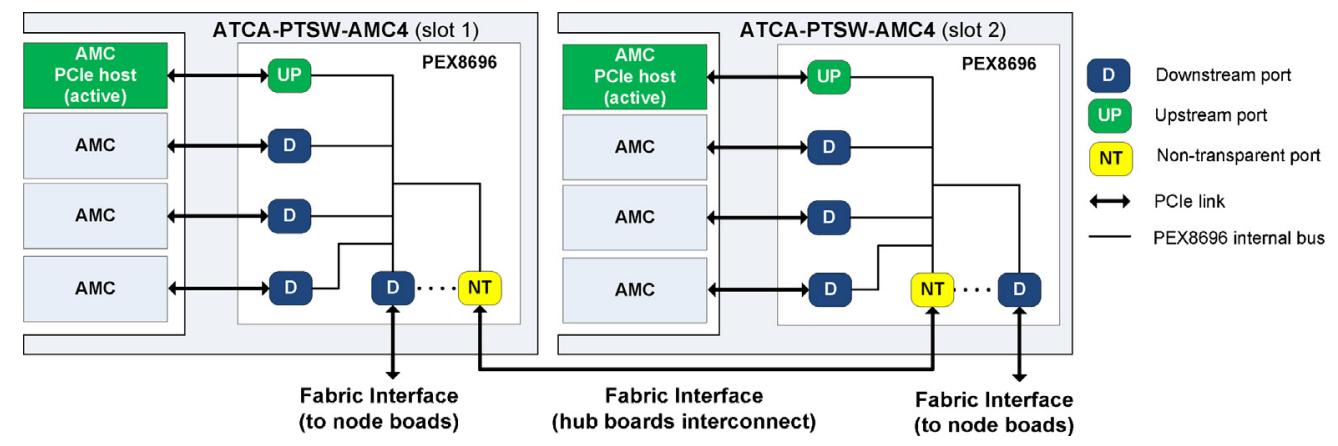

Fig. 3. Active-active redundant host scheme. 


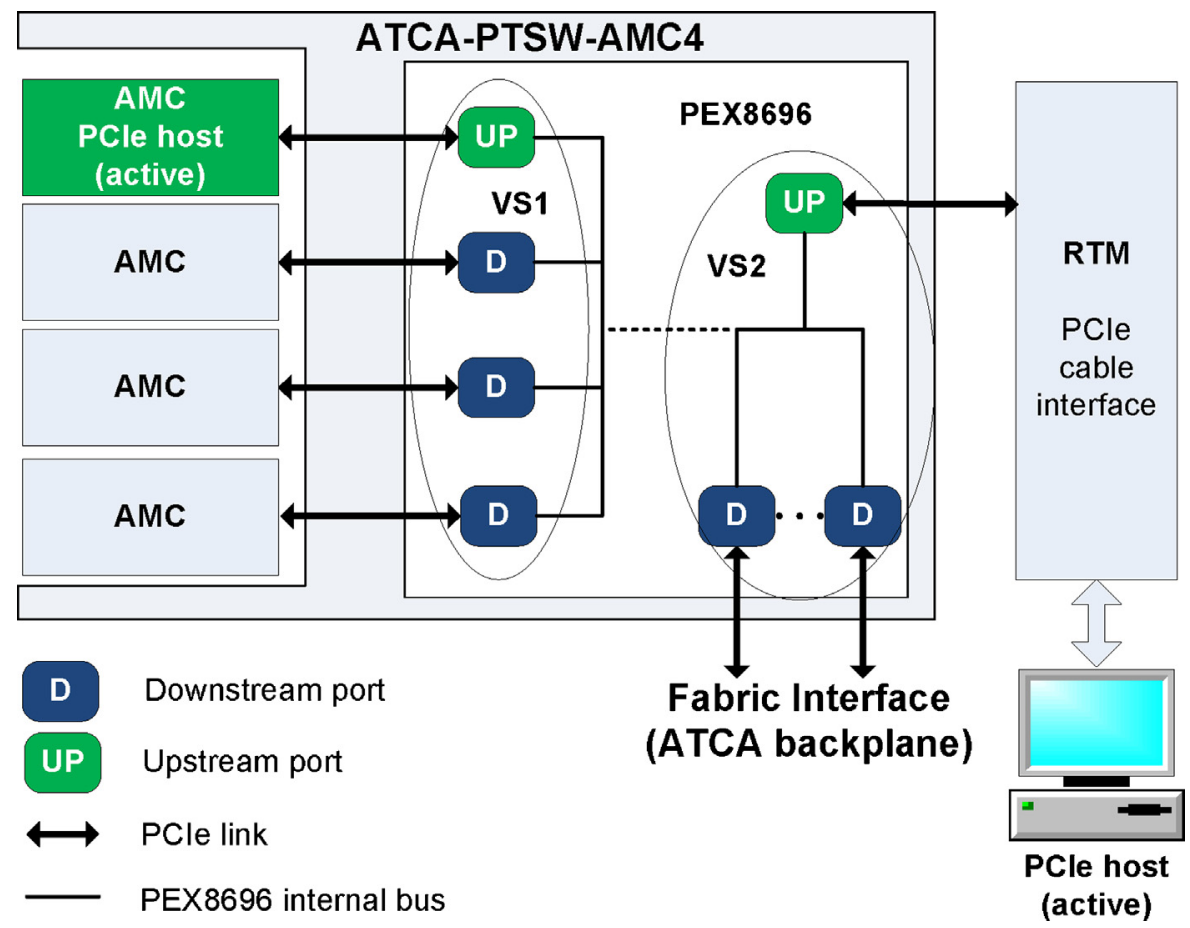

Fig. 4. Extra host added using virtual switch mode.

Switch (VS) mode. VS mode allows a maximum of 8 virtual switches and respective hosts. This mode allows building a modular system where hosts are assigned to different downstream data ports. Hosts may be added to (or removed from) a virtual switch according to processing needs. Alternatively, a host can also be assigned to take over a fault situation. In the application illustrated in Fig. 4, the system has initially one host (AMC) for 3 downstream ports (AMC I/O cards). In order to increase processing power, a second RTM host is assigned to two of the downstream ports, creating two different virtual switches VS1 and VS2.
In Fig. 5 two virtual switches initially operate independently but in case of one of the hosts having a failure, the other host is assigned to take over the unattended downstream ports.

\section{2. $N+1$ redundancy of ATCA modules}

The architecture shown in Fig. 1 offers two basic redundancy scenarios. Firstly, the $2 n$ redundancy of all system modules (replicated ATCA-PSTW-AMC4 "hub" plus ATCA-IO-PROCESSOR “nodes") - half of the modules (for example, odd slot numbers) is active,

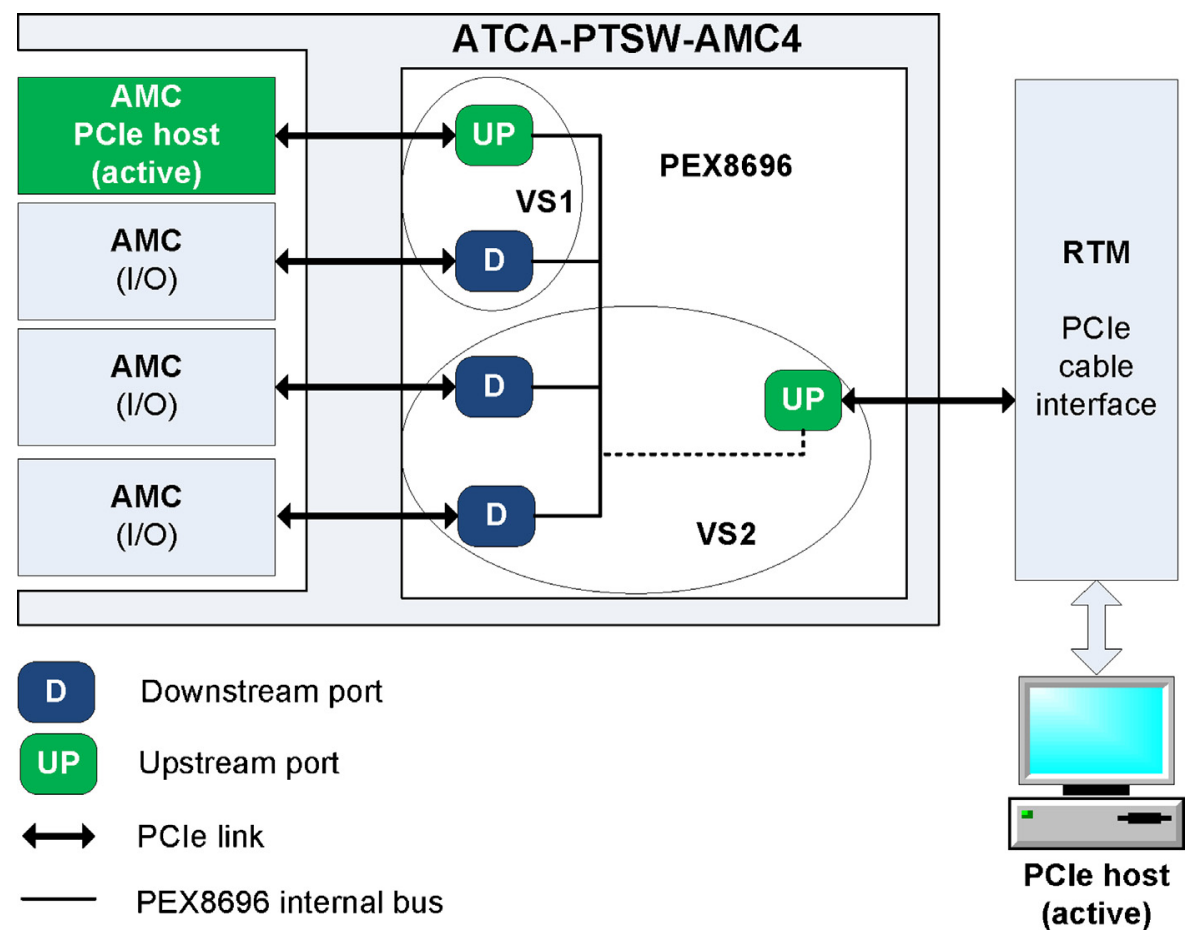

Fig. 5. Host failover multiple-host using virtual switches. 


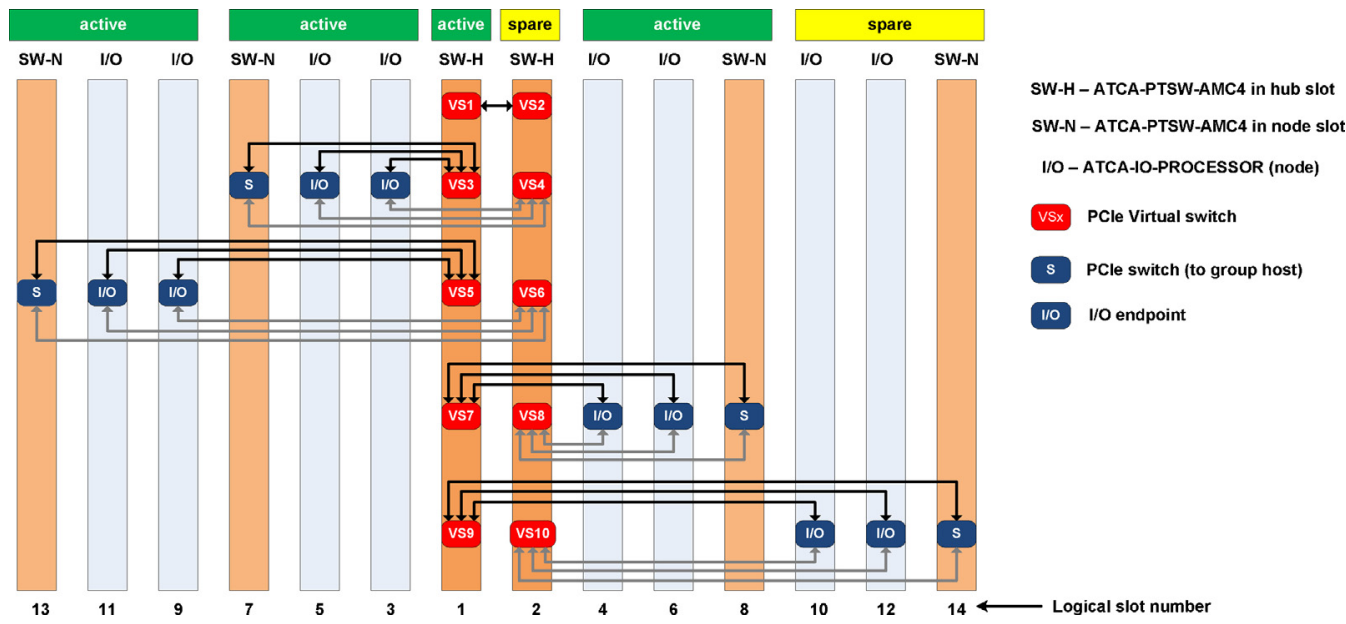

Fig. 6. $N+1$ host and $N+2$ node failover example using virtual switch mode.

and the other half (even slot numbers) are spares. In alternative, one can choose to use all of the $\mathrm{I} / \mathrm{O}$ boards present (without leaving any spares), having just redundant hub modules. This option reduces half the cost per active $\mathrm{I} / \mathrm{O}$ at the (perhaps drastic) expense of its availability. Fig. 6 shows a configuration where a mid-term $N+M$ redundancy scheme can be achieved. The ATCAPTSW-AMC4 modules in hub positions (SW-H) are configured as 5 -virtual-switch hubs. One is active and the other is redundant. VS1 and VS2 interconnect both hubs. The remaining four redundant pairs of virtual switches group four ATCA-PTSW-AMC4 modules in node position (which connect to respective PCle hosts), to four pairs of ATCA-IO-PROCESSOR modules (I/O). In this example, three groups are active and one is a spare. This means that there is one spare hub $(N+1$ redundancy) and two spare node boards $(N+2)$. As upstream/downstream ports of the PEX 8696 may be assigned to any defined VS, other $N+M$ redundancy schemes may be implemented. SW-H and SW-N will differ only on the PEX8696 programming but the hardware module is the same ATCA-PTSWAMC4. No modifications to the I/O cards are required.

\section{Conclusions}

An ATCA-based control and data acquisition subsystem was developed by IPFN to meet ITER requirements. The developed hardware takes advantage of ATCA high-availability qualities. In addition, other solutions were studied on how to optimize redundancy resources. ATCA's fabric channel dual-star topology allows for each module to have its own spare ( $2 n$ redundancy) but that also means that half of the hardware will be not active in a given moment. $N+1$ redundancy schemes may be used to limit the system's cost and keep availability. The bottom line is that this scalable, flexible hardware architecture makes it possible to be quickly reconfigured and implement various redundancy schemes.

\section{Acknowledgments}

This work, supported by the European Communities under the contract of Association between EURATOM/IST, was carried out within the framework of the "European Fusion Development Agreement". Financial support was also received from "Fundação para a Ciência e Tecnologia" and "Programa Operacional Ciência, Tecnologia, Inovação do Quadro Comunitário de Apoio III.” The views and opinions expressed herein do not necessarily reflect those of the European Commission.

\section{References}

[1] Y. Zhang, J. Jiang, Bibliographical review on reconfigurable fault-tolerant control systems, Annual Reviews in Control 32 (2008) 229-252.

[2] Achieving Backplane Redundancy in AdvancedTCA Systems, Radsys, white paper, June 2009.

[3] PCIMG, PICMG ${ }^{\circledR} 3.0$ Revision 3.0 AdvancedTCA ${ }^{\circledR}$ Base Specification, March 2008.

[4] A.J.N. Batista, J. Sousa, C.A.F. Varandas, ATCA digital controller hardware for vertical stabilization of plasmas in tokamaks, Review of Scientific Instruments 77 (10F527) (2006).

[5] D.F. Valcárcel, et al., An ATCA embedded data acquisition and control system for the compass tokamak, Fusion Engineering and Design 84 (June (7-11))(2009) 1901-1904.

[6] M. Correia, et al., ATCA-based hardware for control and data acquisition on nuclear fusion fast control plant systems, IEEE Transactions on Nuclear Science 58 (August (4)) (2011)

[7] B. Gonçalves, et al., ITER fast plant system controller prototype based on ATCA platform, Fusion Engineering and Design (2012) http://dx.doi.org/10.1016/j.fusengdes.2012.04.005.

[8] R.S. Larsen, Life fellow, IEEE, in: PICMG xTCA Standards Extensions for Physics: New Developments and Future Plans, SLAC, Publications, SLACPUB-14182, October 2010. Available from: http://www.slac.stanford.edu/ pubs/slacpubs/14000/slac-pub-14182.pdf

[9] PCIMG, PICMG ${ }^{\circledR} 3.4$ R1.0 Specification - PCI ExpressTM/Advanced Switching for AdvancedTCA ${ }^{\circledR}$ Systems, May 21, 2003.

[10] PCIMG, PICMG ${ }^{\circledR}$ AMC.0 R2.0 - Advanced Mezzanine Card Base Specification, November 2006.

[11] A.J.N. Batista, et al., ATCA/AXIe compatible board for fast control and data acquisition in nuclear fusion experiments, Fusion Engineering and Design (2012), http://dx.doi.org/10.1016/j.fusengdes.2012.05.005.

[12] PEX 8696, PLX Tech, 2011. Available from: http://www.plxtech.com/ products/expresslane/pex8696

[13] Virtex-6 FPGA Family, Xilinx Inc., 2011. Available from: http://www.xilinx com/products/virtex6/

[14] A.P. Rodrigues, et al., Intelligent platform management for fast control plant systems, IEEE Transactions on Nuclear Science 58 (August (4)) (2011). 